\title{
CREATION OF PARAMETRIZED MODEL OF TRANSONIC AXIAL COMPRESSOR FLOW PATH FOR CFD-RESEARCHES
}

\author{
L.N. MARENINA ${ }^{1}$, O.A. SOLOVYEVA ${ }^{2}$, A.A. DROZDOV ${ }^{3}$, YU.B. GALERKIN ${ }^{4}$, V.L. YUSHA \& K. $^{5}$ \\ KABALYK ${ }^{6}$ \\ ${ }^{1,2,3,4}$ National Technology Initiative Center for Advanced Manufacturing Technologies based on the Institute of Advanced \\ Manufacturing Technologies of Peter the Great St. Petersburg Polytechnic University Polytechnicheskaya, 29, St.Petersburg, \\ 195251, Russia \\ ${ }^{5}$ Omsk State Technical University, Russia, Omsk
}

${ }^{6}$ Lodz, University of Technology, Institute of Turbomachinery Wolczanska 219/223 Lodz, Poland

ABSTRACT
Computer programs for primary design based on mathematical pressure losses model and A. Komarov's model of flow
deviation angle are used at the first stage of axial compressors flow path creating. To conduct a gas dynamic study using
CFD methods, it is necessary to create a computational model of the flow path obtained as a result of the design. The
paper describes the main points of creating a parameterized design model of a four-stage axial compressor. The
parameterization is carried out with the next goal: the obtained by the primary design program flow path geometry is
transferred to the ANSYS CFX software package. When copying data, the blades, meridian contour and computational
KEYW are automatically constructed. The resulting model is ready for further gas dynamic calculations.

Received: Jun 09, 2020; Accepted: Jun 29, 2020; Published: Sep 26, 2020; Paper Id.: IJMPERDJUN20201438

\section{INTRODUCTION}

Gas-dynamic calculation of the axial compressor flow path by CFD methods is widely used in design practice. The works [1], [2], [3] show the successful application of computational methods in relation to stator elements of centrifugal compressors, however, CFD modeling of centrifugal compressors in general is still a problem [4], [5], [6]. The positive experience of axial turbines CFD calculations is shown in [7], [8], [9]. Several studies are devoted to the calculation and optimization of axial compressors by CFD methods [10], [11], [12], [13]. When designing a flow path, engineering models and methods play a decisive role [14], [15].

$\mathrm{SPbPU}$ is one of the leading centers for the research and design of compressors for various purposes: the reciprocating compressors [18 - 23], the rotary compressors [24 - 26], as well as turbo compressors [27-32]. The compressor group of the laboratory "Modeling of technological processes and design of power equipment" of the $\mathrm{SPbPU}$ uses in design practice computer programs of its own design, which are based on mathematical pressure losses model and A. Komarov's model of flow deviation angle [16]. Models have been verified, and computer programs have proven their effectiveness in research and design practice [17]. The primary design programs were used to optimize the four-stage low-pressure compressor of the GTE with a pressure ratio $=3.2$.

The purpose of this work was to connect the RCAC primary design program and the Workbench working environment of the ANSYS CFX software package for the possibility of further conducting a numerical gasdynamic study. The primary design program provides information on the flow path meridional dimensions and the 
shape of the impeller and stator blades on 20 cylindrical blade-to-blade surfaces, assesses the components of the pressure loss, the efficiency of the stages and the compressor as a whole, etc. The output data obtained from the RCAC program results is saved in an Excel file in a tabular form. To implement the parameterization, it is necessary to group the data used to build the 3D model in a certain order. For this purpose, a template file is formed in Excel, according to which it is easy to replace data when new geometry is received from the RCAC program.

\section{THE MAIN STAGES OF CREATING A COMPUTATIONAL MODEL}

Fig. 1 shows a simplified outline of a axial compressor stage with an indication of the control sections, as well as a bladeto-blade plane with an indication of the blade angles in the form in which they are presented in the RCAC program.

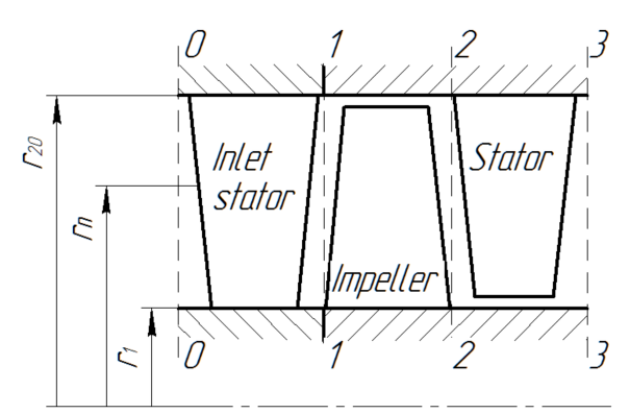

a)

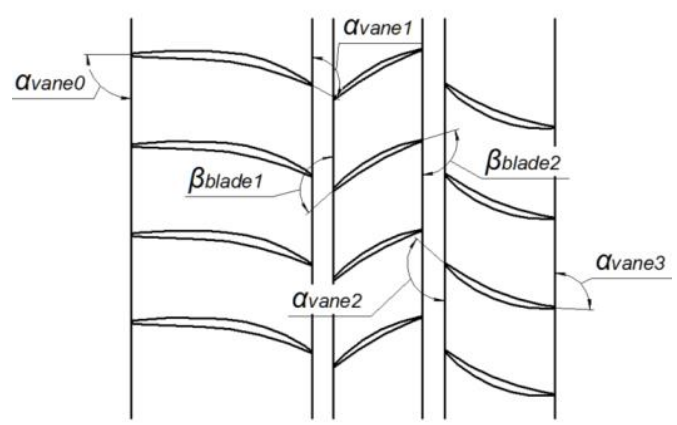

b)

Figure 1: Layout of Control Sections in the Meridional Plane (a); Blade-to-Blade Plane and The Blade Angles (b).

The design of the compressor is carried out on 20 cylindrical blade-to-blade surfaces, thus, to build one blade, it is necessary to construct 20 sketches. Each sketch of impeller blades and stator vanes is determined by the same set of parameters. The inlet stator vane has a more complex geometry, for its construction it is necessary to indicate an additional dimension. The sketches used in the blades construction and the designation of the parameters are shown in Fig. 2.
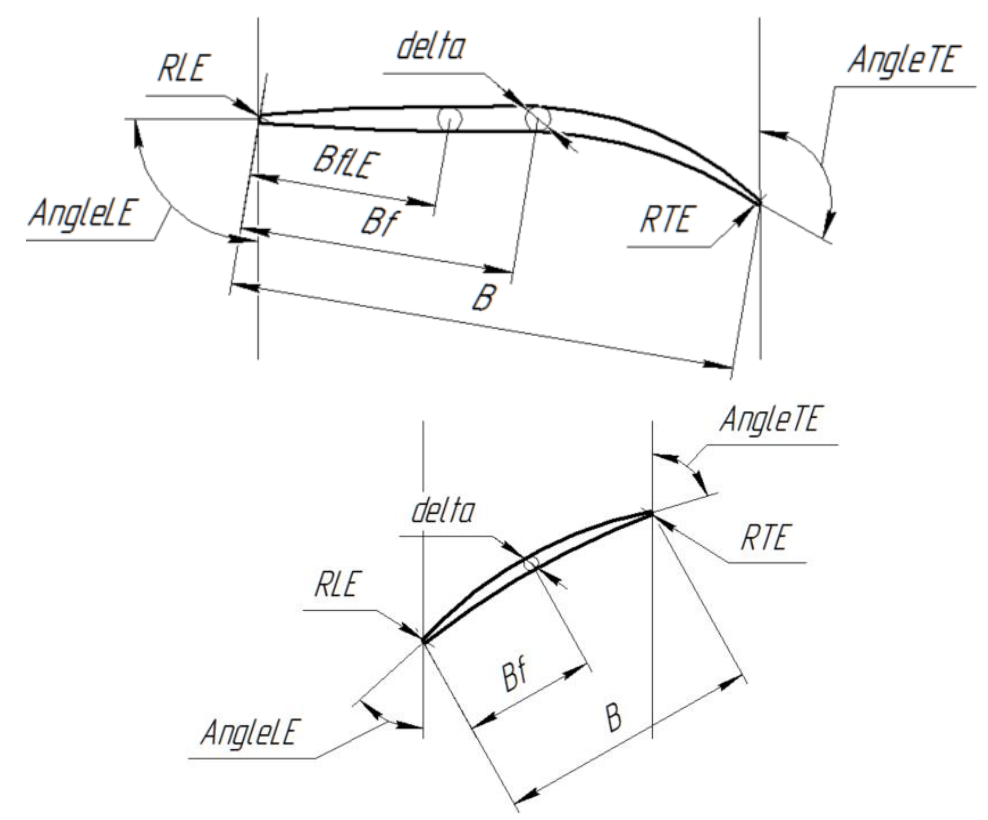

Figure 2: Sketches of Inlet Stator Vane (Top) and Impeller Blade (Bottom). 
The Design Modeler program was used to build the parameterized model. Sketching a vane begins by creating a plane parallel to the $\mathrm{XZ}$ plane. The plane must be located at a distance from the $\mathrm{Z}$ axis equal to the corresponding radius of the cylindrical blade-to-blade surface. This distance is set out as the first Workbench parameter and is designated as R1. Further, in the sketch, auxiliary lines are created, which are necessary to indicate the blade angles. The angles are measured from the $\mathrm{X}$-axis, they are assigned the appropriate names (AngleLE1 and AngleTE1) and they are also set out as parameters (Fig. 3).

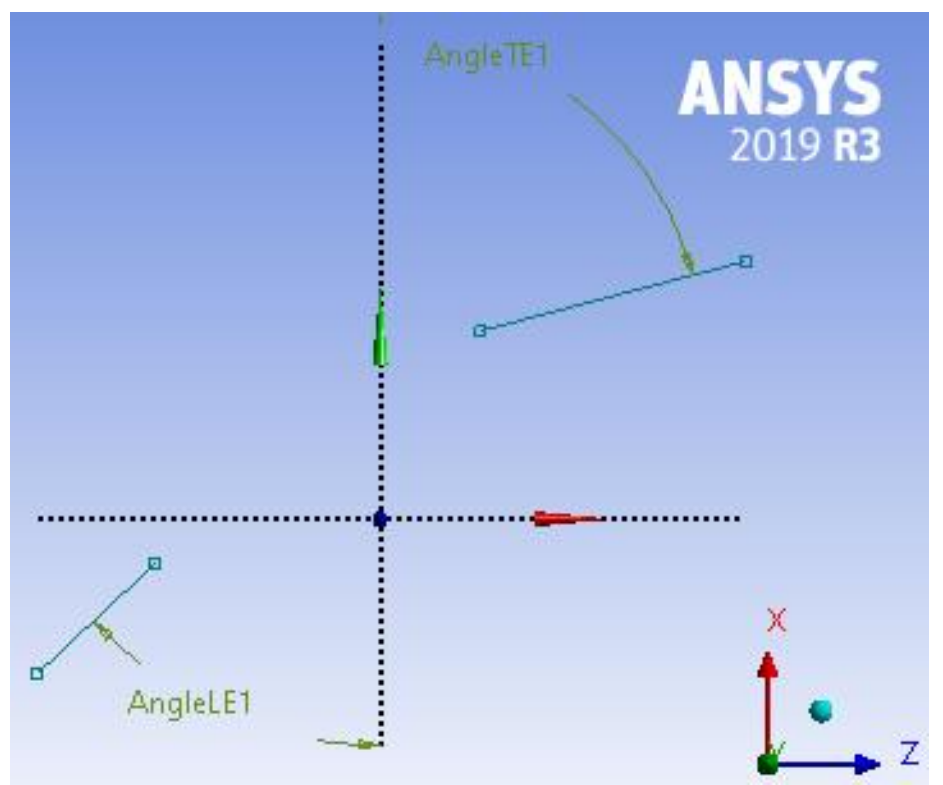

Figure 3: Blade Angles Construction of in the Design Modeler Program.

The camber line of the blade is drawn using the «arc» tool, the condition of tangency to the previously constructed lines is set. A circle centered on the camber line is used to indicate the profile thickness. The diameter of the circle is designated by delta1, set out as a Workbench parameter. Also, to determine the position of the blade relative to the axes, the coincidence of the center of the circle with the center point of the sketch is set. Elements that are finally defined in space are highlighted in blue. Green color means that additional dimensions must be specified (Fig. 4).

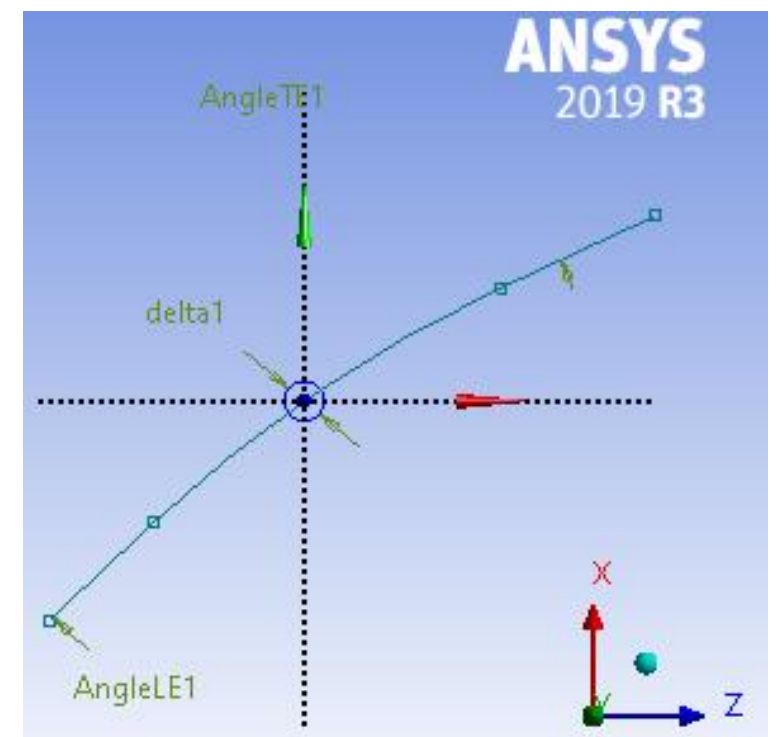

Figure 4: Design Modeler, Creation a Circle that Defines the Profile Thickness. 
At the next stage, the chord of the profile is built, its length is designated as B1. A perpendicular descends from the center of the circle to the chord, the distance from the point of their intersection to the leading edge is denoted by Bf1. This value defines the position of the maximum profile thickness. In the case of a blade, the back and front surfaces of which are constructed using a single arc, Bf1 equals half the chord of the profile (B1). Both values are taken out as parameters of the Workbench, where their relationship is indicated in the parameter table: $\mathrm{P} 3=\mathrm{P} 2 * 0.5$ (Fig. 5), where $\mathrm{P} 1$ ... Pn is the designation of values by continuous numbering.

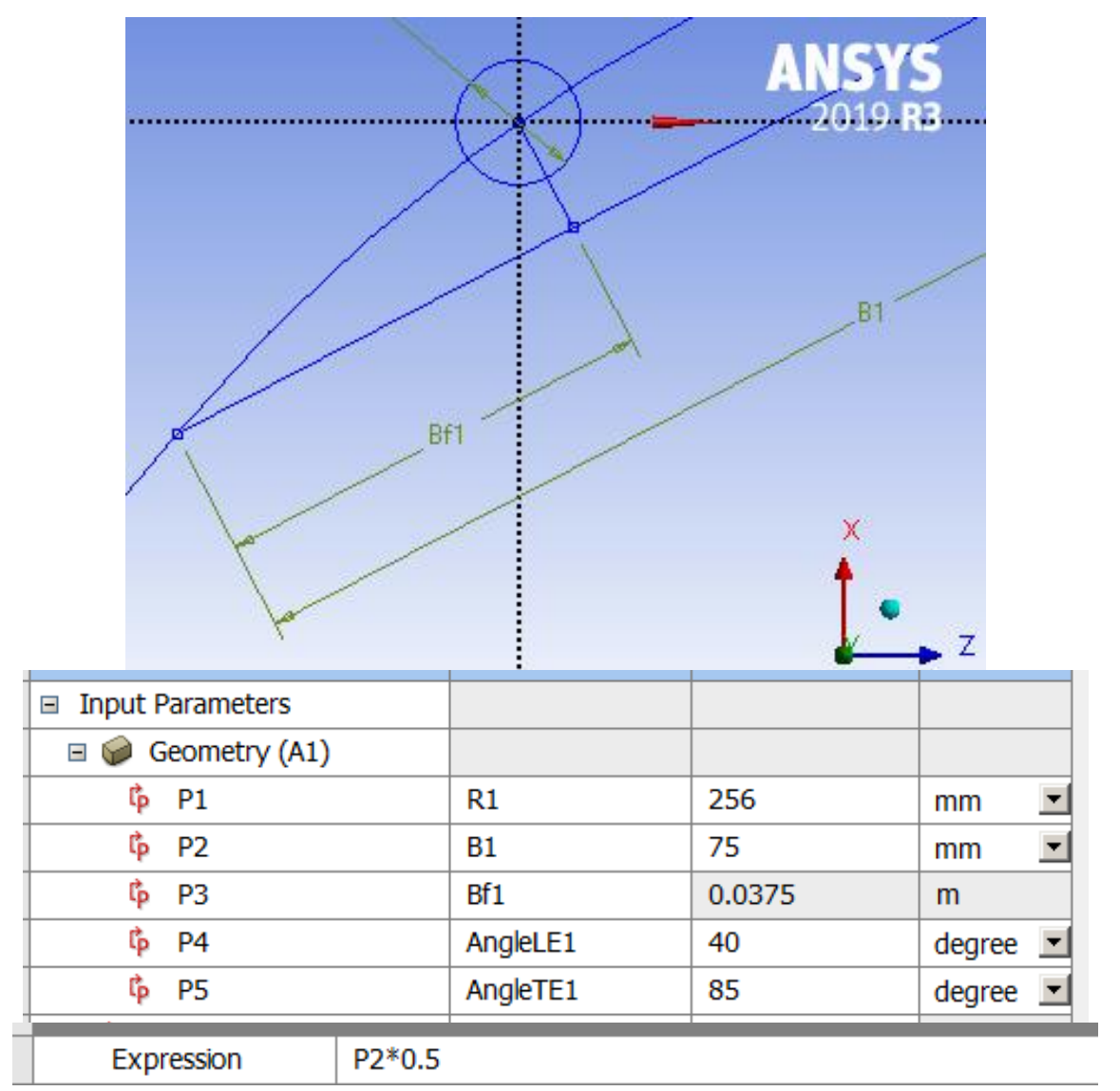

Figure 5: Design Modeler, Setting the Position of the Maximum Profile Thickness.

The next step is to build the outer contour of the blade. To do this, on the sketch, elements of the fillet of the leading and trailing edges are created in the form of arcs, the radii of which are designated RLE1 and RTE1, respectively, and are setting out into the list of parameters. Further, also using the "arc" tool, the front and back surfaces of the blade are constructed, the condition of tangency to the leading and trailing edges and to the circle delta1 is set. The sketch becomes fully defined; no additional dimensions are required. When you change any parameter, the blade is automatically rebuilt. Thus, we have obtained a parametrized sketch of a blade on the first cylindrical blade-to-blade surface, which is suitable for both impeller and stator (Fig. 6). As noted earlier, to construct the inlet stator vane, an additional dimension BfLE is introduced (Fig. 2 top). 


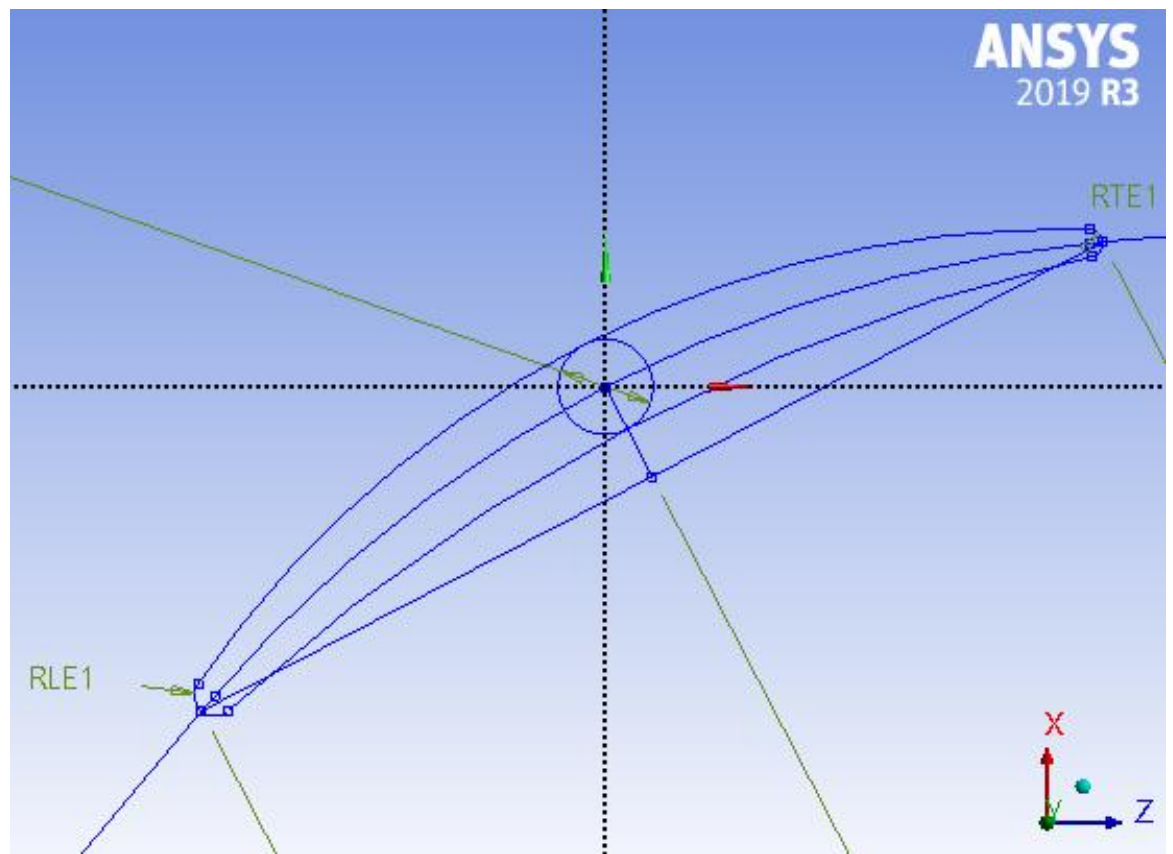

Figure 6: Design Modeler, Parameterized Blade Template for Impeller or Stator.

To describe one sketch of the blades impeller or stator, 8 parameters are used, for inlet stator vane - 9 parameters. In the Excel template file, it is necessary to arrange these parameters in the same order in which they were added to the Workbench parameter table (Figure 7). It is also necessary to pay attention to the fact that in the RCAC program the measuring of the blade angles in the impeller and stator is different (Fig.1 b). With this in mind, the angles for the impeller are recalculated in the Excel template file using the $« 1800-\beta »$ formula Henceforth, the sketches of the blades can be rebuilt by copying data from Excel to the Workbench.

\begin{tabular}{|c|c|c|c|c|c|c|c|c|c|}
\hline \multirow{2}{*}{$\square$ Input Parameters } & & & & & \multirow{2}{*}{$\boxminus$ Inlet stator (G1) } & \multirow[b]{3}{*}{ R1 } & \multirow[b]{3}{*}{-151} & \multirow[b]{3}{*}{$\mathrm{mm}$} & \multirow[b]{3}{*}{ 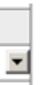 } \\
\hline & & & & & & & & & \\
\hline & & & & & Ґि P654 & & & & \\
\hline $\begin{array}{ll}\text { lp } & \text { P1 } \\
\end{array}$ & R1 & -183.6 & $\mathrm{~mm}$ & $=$ & ҐP P655 & B1 & 114 & $\mathrm{~mm}$ & $\mp$ \\
\hline ЏP P2 & B1 & 66.4 & $\mathrm{~mm}$ & 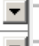 & lp $\quad$ P656 & Bf1 & 68.4 & $\mathrm{~mm}$ & $\nabla$ \\
\hline ¿ $\quad$ P3 & Bf1 & 33.2 & $\mathrm{~mm}$ & $\exists$ & lp P657 & AngleLE1 & 90 & degree & $ت$ \\
\hline ऐ P4 & AngleLE1 & 35.02 & degree & $ت$ & lp $\quad$ P658 & AngleTE1 & 68.314 & degree & 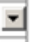 \\
\hline lि P5 & AngleTE1 & 98.782 & degree & 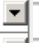 & lp P659 & RLE1 & 0.8 & $\mathrm{~mm}$ & $=$ \\
\hline lp P6 & RLE1 & 0.3 & $\mathrm{~mm}$ & 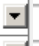 & Ґр $\quad$ P660 & RTE1 & 0.7 & $\mathrm{~mm}$ & $=$ \\
\hline Ґि P7 & RTE1 & 0.3 & $\mathrm{~mm}$ & $=$ & lp P661 & delta1 & 7 & $\mathrm{~mm}$ & $=$ \\
\hline Џp P8 & delta1 & 5 & $\mathrm{~mm}$ & 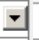 & Ґि P818 & BfLE1 & 45.6 & $\mathrm{~mm}$ & 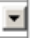 \\
\hline
\end{tabular}

Figure 7: Table of Workbench Parameters: on the Left - Impeller, on the Right - Inlet Stator.

To create sketches in the remaining 19 sections, you must repeat the above steps, assigning the new parameters the number of the corresponding section. To speed up the construction, you can use the "Write script" function, record a sequence of actions and replay them using the "Run script" action. The construction of a three-dimensional blade profile is carried out using a tool Skin/Loft by connecting 20 sketches of the blades into one surface. However, for the correct operation of the "Skin" function, it is necessary to indicate sketches on which there are no additional lines. Also, in order to avoid mistakes, it is recommended to create «Skin» operations separately for the front and back blade surfaces. Taking into account the above, the elements of the blade profile are copied into additional sketches, which will be used when constructing the surface of the blade surfaces (Fig. 8). 


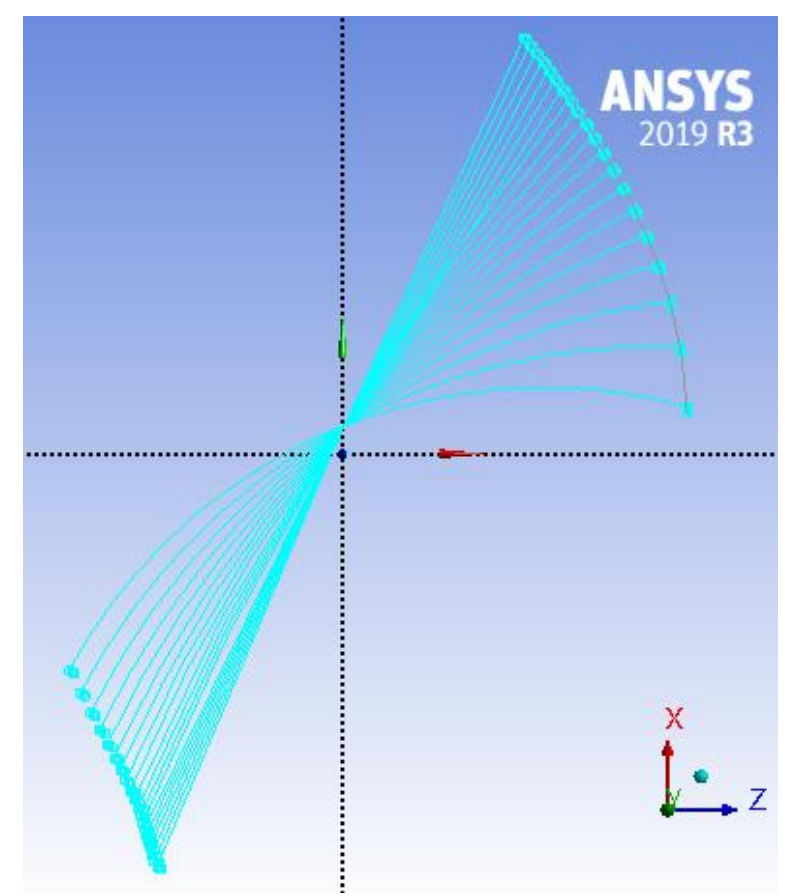

Figure 8: Design Modeler, Construction of the Back Surface of the Blade with a Tool Skin/Loft

To export the finished blade to the TurboGrid, you need to define the position of the hub and shroud. For this purpose, in a separate DesignModeler window, the meridional contour of all four stages of the axial compressor is built and parameterized. The coordinates of the points along the Y axis are denoted as Rnhub and Rnshr for the hub and stator, respectively. The position of points along the $\mathrm{Z}$ axis is specified by the Znhub dimensions and is measured from the $\mathrm{XY}$ plane. The calculation of the Znhub size is described in the Workbench parameter table by a formula that takes into account the axial length of the blades and the gaps sizes between the blade cascades. The general view of the obtained meridional contour is shown in Fig. 9.

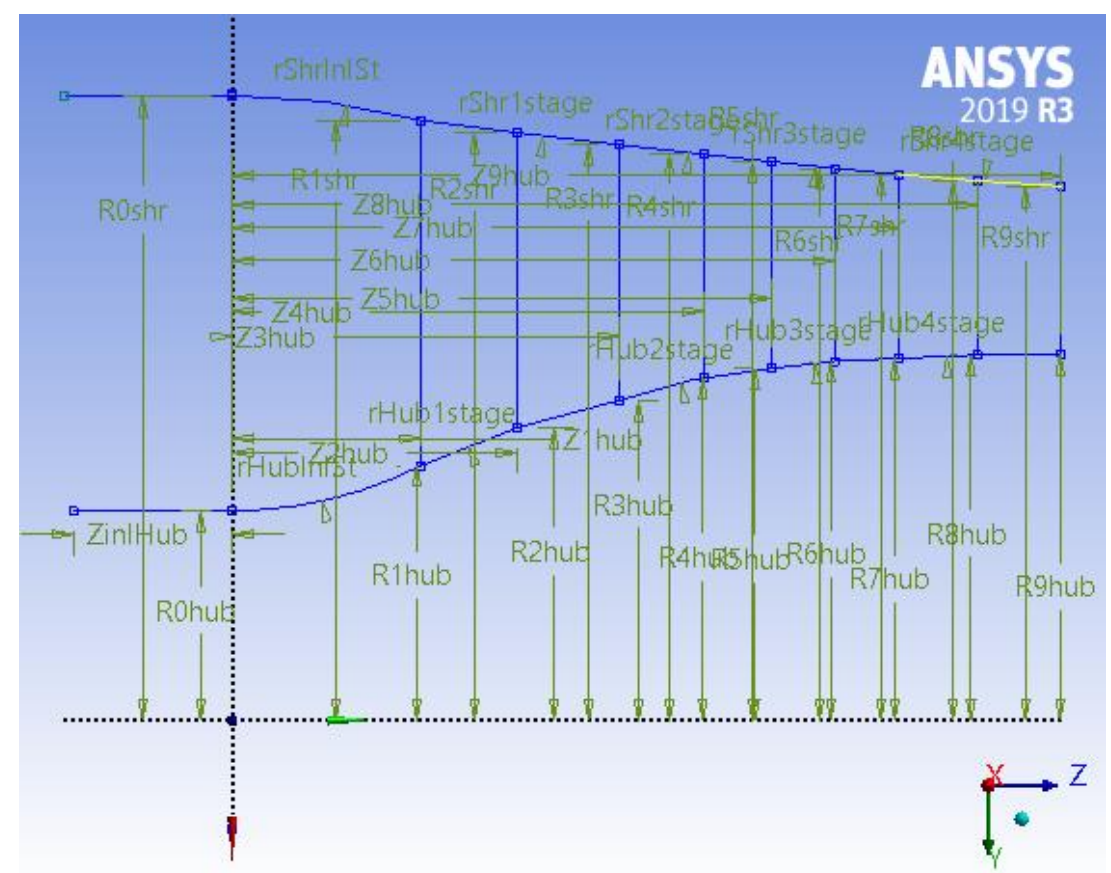

Figure 9: Design Modeler, Parameterized Meridional Contour of a Four-Stage Axial Compressor. 
The radii of curvature of the hub and shroud surfaces obtained from the meridional contour of the compressor are used to construct the meridional contour for each blade separately. For this, a corresponding sketch is created, then a solid body is constructed using the 刺Revolve operation. When creating the "ExportPoints" function, which is required to export geometry to the TurboGrid, it is necessary to select the intersection of this solid with the surface of the finished blade. The final view of the inlet stator, impeller and stator blades of the first stage are shown in Fig. 10.
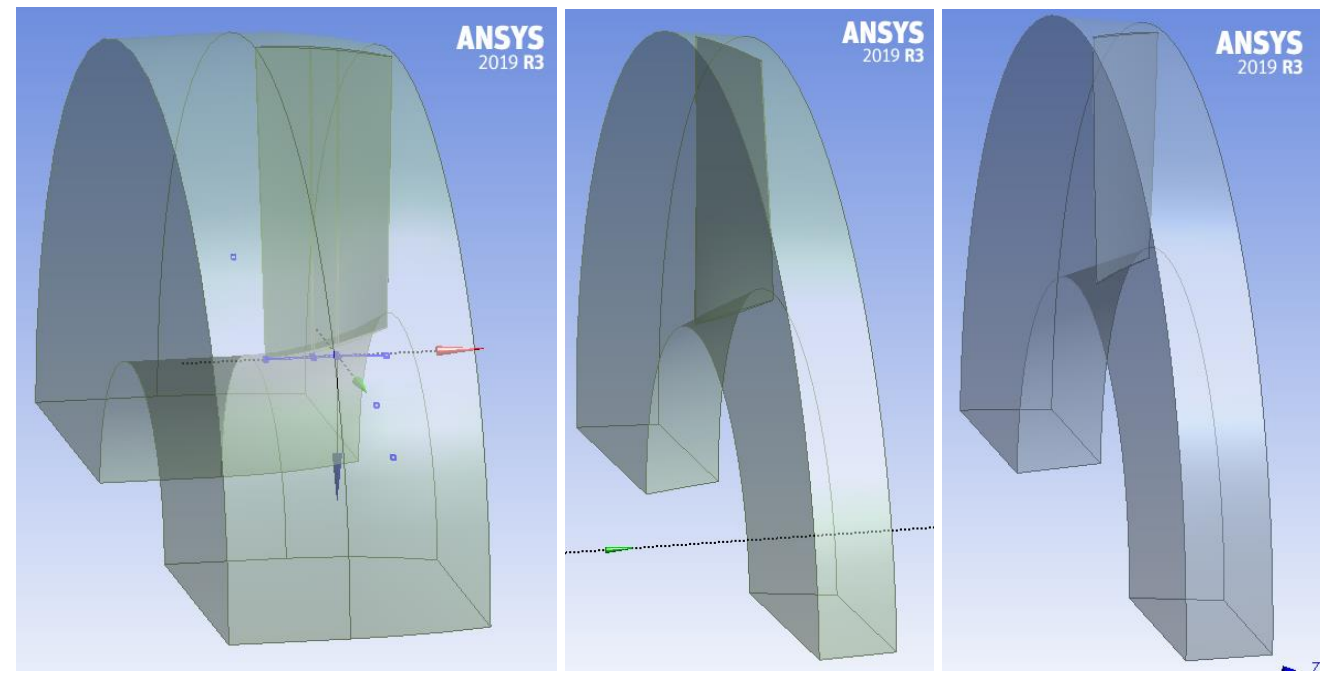
Figure 10: Design Modeler, the Final View of the Inlet Stator Vane (Left), Impeller Blade (Center)
and Stator Vane (Right) of an Axial Compressor First Stage.

TurboGrid program was used to build the grid. The finished structured hexahedral mesh consists of about 400,000 elements for one blade row. For further gas-dynamic calculation, it is assumed that the SST (Shear Stress Transport) turbulence model will be used, which requires values of $y+<10$. With this in mind, the size of the first near wall element is about $0.015 \mathrm{~mm}$.

At the next stage, it is necessary to unload the finished meshes into the CFX-Pre program, where the boundary conditions for all surfaces are set in a semi-automatic mode. At the inlet and outlet surfaces, the walls are assumed to be hydraulically smooth. The Stage interface is used between the stationary and rotating elements. Total pressure and total temperature are set at the inlet, and static pressure at the outlet. The general view of the obtained design model of a fourstage axial compressor is shown in Fig. 11. 


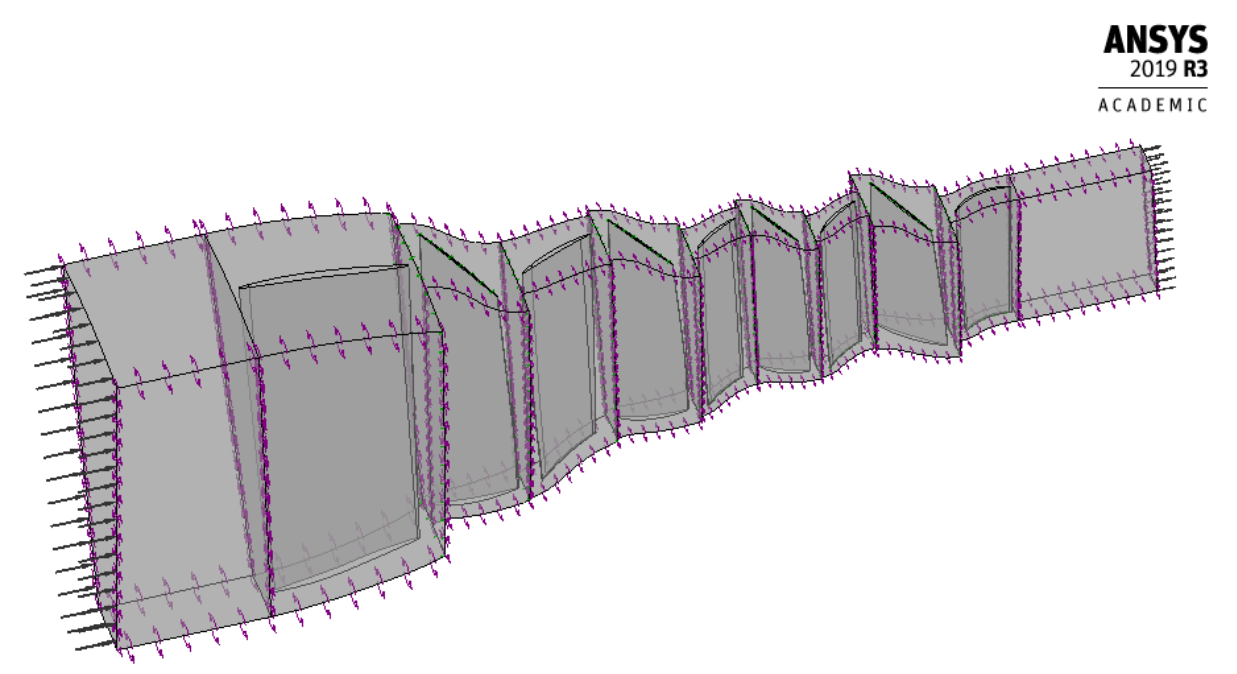

Figure 11: CFX-Pre, General View of the Finished Design Model of a Four-Stage Axial Compressor.

\section{CONCLUSIONS}

As a result of the work done, a parameterized design model of an axial compressor was obtained. The parameterization was carried out in such a way that when designing the flow path in the RCAC program, the obtained new geometry was easily transferred to the ANSYS CFX software package. This makes it possible to link two tools for calculating and designing axial compressors into a single module, which allows, at the initial design stage, to determine the most suitable version of the compressor for the given dimensions and estimate its parameters, and at the stage of CFD calculations to fine-tune it and specify the dimensions of the flow path elements. When copying data from an Excel template file to the Workbench parameter table, the blades, meridian contour and computational grids are automatically rebuilt. The resulting model is completely ready for further gas-dynamic calculations.

\section{ACKNOWLEDGEMENTS}

The calculations were performed using the supercomputer of the center "Polytechnic" SPbPU. The study was funded by a grant from the Russian science Foundation (project No. 18-79-10165).

\section{REFERENCES}

1. Galerkin Y., Drozdov A., Solovyeva O. Vaneless diffuser for low flow rate centrifugal compressor stage. - Proceedings of 13th European Conference on Turbomachinery Fluid dynamics \& Thermodynamics. - Paper ID: ETC2019-329. - ETC13, Lausanne, Switzerland. - April 8-12, 2019

2. Galerkin Yu.B., Drozdov A.A., Solovyeva O.A. Features of the operation of vaneless diffusers of centrifugal compressor stages with different meridional shapes. Proceedings of the 16th International Scientific and Technical Conference on Compressor Engineering. - SPb. - Volume 1. - 2014. - p. 171-178.

3. Marenina L., Galerkin Y., Soldatova, K. Computational fluid dynamics application for analysis of centrifugal compressor stage stator part. - International Journal of Mechanical Engineering and Robotics Research. - 7 (6), pp. 656-661. DOI: 10.18178/ijmerr.7.6.656-661

4. Borovkov A.I., Voinov I.B., Nikitin M.A., Galerkin Yu.B., Rekstin A.F., Drozdov A.A. Ex-perience of performance modeling the single-stage pipeline centrifugal compressor. - Oil and Gas Engineering- AIP Conf. Proc. 2141. - Omsk. - Russia. - 2019, 030051-030051-10, https://doi.org/10.1063/1.5122101 
5. Borovkov A.I., Voinov I.B., Galerkin Yu.B., Drozdov A.A., Soldatova K.V. Experimental characteristic simulation for two-stage pipeline centrifugal compressor. International Conference on Compressors and their Systems 2019. IOP Conf. Series: Materials Science and Engineering 604 (2019) 012052. doi:10.1088/1757-899X/604/1/012052

6. Borovkov A., Voinov I., Galerkin Y., Nikiforov A., Nikitin M., Solovyeva O. and Kabalyk K. Issues of gas dynamic characteristics modeling: a study on a centrifugal compressor model stage. - E3S Web of Conferences 140, 06003 (2019). International Scientific Conference on Energy, Environmental and Construction Engineering (EECE-2019) https://doi.org/10.1051/e3sconf/201914006003

7. Rakov, G.L., Rassokhin, V.A., Zabelin, N.A., Olennikov S.Y., Sebelev A.A., Sukhanov, A.I., Schislyaev, S.M.A low emission axial-flow turbine for the utilization of compressible natural gas energy in the gas transport system of Russia. International Journal of Environmental and Sci-ence Education. 2016. Vol. 11, № 18, pages 11721-11733

8. Smirnov, M.V., Sebelev, A.A., Zabelin, N.A., Kuklina, N.I. Effects of hub endwall geome-try and rotor leading edge shape on performance of supersonic axial impulse turbine. Part I. 12th European Conference on Turbomachinery Fluid Dynamics and Thermodynamics, ETC 2017

9. Sebelev, A.A., Smirnov, M.V., Borovkov, A.I., Kuklina, N.I., Rakov, G.L.Effects of hub endwall geometry and rotor leading edge shape on performance of supersonic axial impulse turbine. Part II: Method validation and final results. 13th European Turbomachinery Conference on Turbomachinery Fluid Dynamics and Thermodynamics, ETC 2019

10. 1. J. Zhang, Z. Zhou, H. Cao, Q. Li Aerodynamic design of a multi-stage industrial axial compressor. Advances in Engineering Software $116(2018)$ 9-22

11. L. Zhihui, L. Yanming, Optimization of rough transonic axial compressor, Aerosp. Sci. Technol. (2018)

12. M. S. Aftab, F. Ali, M. Aadil Khan, K. Parvez Design and analysis of a five stage axial flow compressor. 2017 th International Conference on Aerospace Science and Engineering, ICASE 20176 June 2018, Pages 1-9

13. E. Marchukov, I. Egorov, G. Popov, O. Baturin, E. Goriachkin, Y. Novikova Optimization of a three spool axial compressor to increase the efficiency of a gas turbine engine. IOP Conf. Series: Materials Science and Engineering 604 (2019) 012048

14. Galerkin Yu.B., Rekstin A.F., Soldatova K.V., Drozdov A.A., Solovyova O.A., Semenovskiy V.B. The state of the engineering method for optimal gas dynamic design and calculation of the characteristics of centrifugal compressors. Part 1. Compressor technology and pneumatics. - 2019. - No. 4. - p. 3 - 10.

15. Galerkin, Y., Rekstin A., Soldatova K., Drozdov A., Popov Y.A. Development the scientific turbomachinery school LPI-SpbPu, the results of cooperation with industry. 17 international scientific and technical conference on compressors. p. 19-29 (2017)

16. Komarov A.P. Investigation of flat compressor cascades. / Blade machines and jet devices, Sum. articles, Issue 2. Moscow: Mechanical Engineering 1967 p. 67-110.

17. Popov Yu.A. Improvement and analysis of the flow path of axial compressors and stages using the results of vane cascade testing. [text]: dis ... cand. tech. sciences. / Yu.A. Popov; SPbSPU. - SPb., 2010.

18. Kotlov A.A., Maksimenko I.A. Mathematical modelling the seasonal load of opposite compressors. - Oil and Gas Engineering (OGE-2018). - AIP Conf. Proc. 2007. - Omsk. - Russia. - 2018. 030053-1-030053-9; https://doi.org/10.1063/1.5051914

19. Kotlov A.A. A reciprocating compressor operation in a net with pulsating pressure. - Oil and Gas Engineering (OGE-2018). AIP Conf. Proc. 2007. - Omsk. - Russia. - 2018. - 030038-1-030038-6; https://doi.org/10.1063/1.5051899 
20. Andrey Kotlov, Leonid Kuznetsov and Boris Hrustalev Influence of compressible medium on the operation of a reciprocating compressor. - MATEC Web Conf. Volume 245, 2018. - International Scientific Conference on Energy, Environmental and Construction Engineering (EECE-2018). DOI 10.1051/matecconf/201824504009

21. Kotlov A.A., Kuznetsov L.G., Burakov A.V. Analysis of reciprocating booster compressor performance by mathematical modelling methods. - Oil and Gas Engineering- AIP Conf. Proc. 2141. - Omsk. - Russia. - 2019, 030012-030012-9, https://doi.org/10.1063/1.5122062

22. Kotlov A.A. Calculation and selection of parameters of the ring valve of reciprocating compressor. - Oil and Gas EngineeringAIP Conf. Proc. 2141. - Omsk. - Russia. - 2019, 030020-030020-10, https://doi.org/10.1063/1.5122070

23. Kotlov A.A. Research of two-stage reciprocating compressor by methods of physical and mathematical experiments. - Oil and Gas Engineering- AIP Conf. Proc. 2141. - Omsk. - Russia. - 2019, 030021-030021-10, https://doi.org/10.1063/1.5122071

24. A. A. Kotlov, I. A. Maksimenko and Y. L. Kuznetsov. The influence of profile geometric parameters on characteristics of rotorgearing compressor. IOP Conf. Series: Materials Science and Engineering 425 (2018) 012012 doi:10.1088/1757$899 X / 425 / 1 / 012012$.

25. A. A. Kotlov. Influence of suction port parameters on integral characteristics of screw-type compressor. IOP Conf. Series: Materials Science and Engineering 425 (2018) 012014 doi:10.1088/1757-899X/425/1/012014.

26. Andrey Kotlov, Leonid Kuznetsov and Boris Hrustalev Investigation of the influence of the number of vanes on the performance of a rotary vane compressor. - MATEC Web Conf. Volume 245, 2018. - International Scientific Conference on Energy, Environmental and Construction Engineering (EECE-2018). DOI 10.1051/matecconf/201824504008

27. Soldatova K. Modeling of small-size turbocharger compressors' performance curves. International Conference on Compressors and their Systems. London. City University. - UK. -2013. - P. - 707-716

28. Soldatova K. CFD study of leakage flows in shroud cavities of a compressor impeller. International Conference on Compressors and their Systems. London. City University. - UK. - 2017. - http://iopscience.iop.org/article/10.1088/1757899X/232/1/012046. - DOI: 10.1088/1757-899X/232/1/012045

29. Nikiforov A., Popova D., Soldatova K. A network application for modeling a centrifugal compressor performance map. International Conference on Compressors and their Systems. London. City University. - UK. - $2017 . \quad$ http://iopscience.iop.org/article/10.1088/1757-899X/232/1/012046. - DOI: 10.1088/1757-899X/232/1/012046.

30. Rekstin A., Popova E., Ucehovscy A. Centrifugal compressor stages efficiency analysis by means of the approximate algebraic equations. - Oil and Gas Engineering (OGE-2018). - AIP Conf. Proc. 2007. - Omsk. - Russia. - 2018. - 030036-1-030036-11; https://doi.org/10.1063/1.5051897

31. Galerkin, Yu. B. Turbocompressors. Workflow, calculation and design of the flow path. [text] / Yu. B. Galerkin//Information and publishing center KHT. - 2010. P. 596 p.

32. Galerkin, Yu. B. Modeling of the working process of industrial centrifugal compressors. Scientific bases, stages of development, current state. / Yu. B. Galerkin, K. V. Soldatova // Saint Petersburg: Polytechnic University Publishing house, 2011. - $328 p$. 